\title{
Linear response, fluctuation-dissipation, and finite-system-size effects in superdiffusion
}

\author{
Aljaž Godec ${ }^{1,2, *}$ and Ralf Metzler ${ }^{1,3, \dagger}$ \\ ${ }^{1}$ Institute for Physics \& Astronomy, University of Potsdam, 14476, Potsdam-Golm, Germany \\ ${ }^{2}$ National Institute of Chemistry, 1000, Ljubljana, Slovenia \\ ${ }^{3}$ Physics Department, Tampere University of Technology, 33101, Tampere, Finland \\ (Received 23 April 2013; revised manuscript received 27 June 2013; published 15 July 2013)
}

\begin{abstract}
Lévy walks (LWs) are a popular stochastic tool to model anomalous diffusion and have recently been used to describe a variety of phenomena. We study the linear response behavior of this generic model of superdiffusive LWs in finite systems to an external force field under both stationary and nonstationary conditions. These finite-size LWs are based on power-law waiting time distributions with a finite-time regularization at $\tau_{c}$, such that the physical requirements are met to apply linear response theory and derive the power spectrum with the correct short frequency limit, without the introduction of artificial cutoffs. We obtain the generalized Einstein relation for both ensemble and time averages over the entire process time and determine the turnover to normal Brownian motion when the full system is explored. In particular, we obtain an exact expression for the long time diffusion constant as a function of the scaling exponent of the waiting time density and the characteristic time scale $\tau_{c}$.
\end{abstract}

DOI: 10.1103/PhysRevE.88.012116

PACS number(s): 05.40.Fb, 02.50.Ey, 87.10.Mn, 89.75.Da

\section{INTRODUCTION}

Suppose you follow a tracer particle in an intermittent chaotic system. While the particle will move ballistically in laminar regions, it will be trapped intermittently around sticky islands [1]. Typically the resulting motion is superdiffusive, with a mean squared displacement (MSD) of the form $\left\langle\mathbf{r}^{2}(t)\right\rangle \simeq t^{3-\alpha}$ and $1<\alpha<2$ [2,3]. Such laminar-localized motion of tracer particles was observed in a rotating annulus in the classical experiment by Solomon, Weeks, and Swinney with $\alpha=1.35$ [4]. A similar behavior was observed for particle motion encoded in dynamic maps [5,6]. Processes of this kind are statistically described by Lévy walks (LWs), a variant of Scher-Montroll continuous time random walks (CTRWs) with a spatiotemporal coupling between waiting times and jump lengths [3,7-9]. This coupling ensures that the walker moves with a finite velocity and thus possesses a finite MSD while developing a fractal trajectory with long-tailed distribution of relocation lengths [10].

Along with recent advances in experimental techniques LWs have undergone a revival. The most remarkable development is the possibility to manufacture disordered optical materials, in which light beams travel superdiffusively and follow LW patterns [11]. This experiment could be the basis for new opaque optical materials or lasers, and form the foundation for the understanding of medical tissue imaging. The characteristics of LW superdiffusion were also detected for photon transport in hot vapors of rubidium atoms due to inelastic scattering [12]. These results offer dramatic new insight in such systems as stars, gas lasers, discharges, or hot plasmas. LW characteristics also underlie the dynamics of on-off blinking dynamics of the luminescence in quantum dots, or molecular emitters such as dyes or biopolymers [13].

\footnotetext{
*aljaz.godec@ki.si

†rmetzler@uni-potsdam.de
}

Concurrently, LWs are being identified as the governing process in an ever increasing number of random search and motion patterns of animals [14] and humans [15]. Specifically, LWs describe exhaustive tracking data of marine predators [16], the spreading of mussels [17] and lice [18], or the flights of bats [19]. A central advantage of the LW search strategy is that its scale-free nature makes it robust against environmental changes [20].

LWs are by now a well established and efficient tool in the description of dynamic systems. However, several crucial questions on their fundamental physical behavior remain elusive. One central issue is how to include the effects of the crucial finiteness of the systems under consideration: finite territories of animals or the maximum depth to which sharks can dive, the size of disordered optical materials, hot vapors, and of medical tissue samples, or the finite distance between stable islands in a chaotic sea. The other is the exact treatment of the essential physical response behavior to external forces as well as the power spectrum. Thus, what is the effect of the directional rotation of the annulus, an asymmetry in the dynamic maps, a nutrition gradient on the sea floor for mussels, an underwater current or wind affecting the motion of fishes or birds, or an optical anisotropy in Lévy glasses?

Here we take recent results on the $1 / f$ noise behavior of LWs [21], the Einstein relation of LWs and their ergodic behavior [22,23] one step further: the consideration of a finite but otherwise arbitrary system size allows us to exactly derive the linear response behavior of LWs to a constant or harmonic external force based on the Kubo theory. We derive a generalized Einstein relation, which is eventually replaced by the classical Einstein relation at long times. We also obtain the power spectrum of LWs and show that its low-frequency limit consistently converges to the long-time diffusion constant, for which we obtain an exact expression. Our approach is universal in the sense that it leads to results valid over the entire time evolution of the process including the turnovers between different regimes, and it holds for ballistic, superdiffusive, and normally diffusive motion on an equal footing. 


\section{RESULTS AND DISCUSSION}

\section{A. Time-averaged mean squared displacement}

In the velocity model an LW moves to the left or right with fixed speed $v$ [3]. It randomly changes directions after the expiration of a waiting time $t$. These waiting times are independent random variables with probability density $\psi(t)$ [24]. The joint probability density for a jump of length $x$ given the waiting time $t$ is $\Psi(x, t)=\frac{1}{2} \psi(t) \delta(|x|-v t)$, and thus confines the free paths of the LW within an expanding horizon at positions $v t$ [24]. For $\psi(t)$ we choose the form

$$
\psi(t)=\frac{\tau_{c}^{\alpha}}{\Gamma\left(-\alpha, \tau_{c}^{-1}\right)(1+t)^{1+\alpha}} \exp \left(-\frac{1+t}{\tau_{c}}\right),
$$

turning over from the power-law form $\psi(t) \simeq(1+t)^{-(1+\alpha)}$ for $t \ll \tau_{c}$ to an exponential wing at $t \gg \tau_{c}$ [25] and mean sojourn time

$$
\bar{\tau} \equiv \int_{0}^{\infty} \tau \psi(\tau) d \tau=\frac{\tau_{c} \Gamma\left(1-\alpha, \tau_{c}^{-1}\right)}{\Gamma\left(-\alpha, \tau_{c}^{-1}\right)}-1 .
$$

For a finite system the turnover time $\tau_{c}$ is related to the system size $L$ via the scaling $\tau_{c} \simeq L /|v|$, and is thus a natural physical parameter. While $\tau_{c}$ can, in principle, be chosen arbitrarily large and thus the power-law form of $\psi(t)$ dominates the LW, the presence of $\tau_{c}$ ensures that the process eventually diffuses normally and that it fulfills the physical conditions to apply linear response theory. Such finite-size effects were indeed observed empirically for LW dynamics in optical materials [11], of tracers in the rotating annulus [4], and for animal motion $[18,19]$.

Our derivations are based on the velocity autocorrelation $C_{v}(t)=\lim _{t^{\prime} \rightarrow \infty}\left|t^{\prime}-t\right|^{-1} \int_{0}^{\left|t^{\prime}-t\right|} v\left(t^{\prime \prime}\right) v\left(t^{\prime \prime}+t\right) d t^{\prime \prime}$. From the renewal approach $[5,22]$ we obtain

$$
C_{v}(t)=v^{2} \frac{\Gamma\left(1-\alpha, \frac{1+|t|}{\tau_{c}}\right)-\left(\frac{1+|t|}{\tau_{c}}\right) \Gamma\left(-\alpha, \frac{1+|t|}{\tau_{c}}\right)}{\Gamma\left(1-\alpha, \tau_{c}^{-1}\right)-\tau_{c}^{-1} \Gamma\left(-\alpha, \tau_{c}^{-1}\right)}
$$

with the upper incomplete $\Gamma$ function $\Gamma(a, z)$ [26]. In Fig. 1, Eq. (3) agrees perfectly with numerical simulations. Via the Kubo formula [27], from Eq. (3) we obtain the time-averaged MSD

$$
\overline{\delta^{2}(\Delta)}=\lim _{T \rightarrow \infty} \frac{1}{T-\Delta} \int_{0}^{T-\Delta}[x(t+\Delta)-x(t)]^{2} d t
$$

in terms of the lag time $\Delta$ and the measurement time $T$. It was noted previously [28] that $\overline{\delta^{2}(\Delta)}$ is equivalent to the stationaryensemble average $\left\langle x^{2}(t)\right\rangle_{s}$ of a system prepared at $t=-\infty$ (i.e., independent of its initial condition) and recorded from $t=0$. The exact result becomes

$$
\overline{\delta^{2}(\Delta)}=2 \frac{\tau_{c}^{2} \sum_{k=2}^{3} \frac{(-1)^{k}}{k !}\left\{\left(\frac{1+\Delta}{\tau_{c}}\right)^{k} \Gamma\left(3-k-\alpha, \frac{1+\Delta}{\tau_{c}}\right)+\left(\frac{1+\Delta}{\tau_{c}}\right)^{3-k}\left[\Gamma\left(k-\alpha, \tau_{c}^{-1}\right)-\Gamma\left(k-\alpha, \frac{1+\Delta}{\tau_{c}}\right)\right]\right\}+\Theta}{\Gamma\left(1-\alpha, \tau_{c}^{-1}\right)-\tau_{c}^{-1} \Gamma\left(-\alpha, \tau_{c}^{-1}\right)},
$$

where we have introduced the auxiliary abbreviation

$$
\begin{aligned}
\Theta= & (1+\Delta)\left\{\tau_{c}^{1+\alpha} e^{-1 / \tau_{c}}-\Gamma\left(-\alpha, \tau_{c}^{-1}\right)\left(\alpha \tau_{c}+1 / 2\right)\right\} \\
& -\Gamma\left(-\alpha, \tau_{c}^{-1}\right)\left(\alpha \tau_{c} / 2-1 / 3\right) .
\end{aligned}
$$

The scaling behavior at intermediate times $1 \ll t \ll \tau_{c}$ follows from the expansion

$$
\Gamma(a, z)=\Gamma(a)-\sum_{k=0}^{\infty}(-1)^{k} \frac{z^{k+a}}{k !(k+a)}
$$

and the expansion of Eq. (2)

$$
\bar{\tau} \sim \begin{cases}\frac{\tau_{c}^{1-\alpha} \Gamma(2-\alpha)}{1-\alpha\left(1-\tau_{c}^{-1}\right)}, & 0<\alpha<1, \\ \frac{2-\alpha\left(1-\tau_{c}^{-1}\right)}{(2-\alpha)\left[\alpha\left(1-\tau_{c}^{-1}\right)-1\right]}, & 1<\alpha<2 .\end{cases}
$$

The intermediate scaling behavior reads

$$
\left\langle\overline{\delta^{2}(\Delta)}\right\rangle \sim \begin{cases}t^{2}, & 0<\alpha<1, \\ 2 \zeta t^{3-\alpha}, & 1<\alpha<2,\end{cases}
$$

where $\zeta=1 /(3-\alpha)(2-\alpha)$. These are the familiar ballistic and subballistic diffusion behaviors of LWs, for diverging $(0<\alpha<1)$ and finite $(1<\alpha<2)$ mean waiting times $[3,5,24]$. As expected, beyond $\tau_{c}$ the motion becomes normally diffusive $\overline{\delta^{2}(\Delta)} \sim D\left(\alpha, \tau_{c}\right) \Delta$. The long-time diffusion constant is analyzed below. The right panel of Fig. 1 shows excellent agreement between our rigorous theoretical result (5) and simulations. The anomalous to normal diffusion turnover indicates a separation of time scales, see below.

We emphasize that even for long time series, in which the particle traverses the system repeatedly, we observe an anomalous scaling of $\overline{\delta^{2}}$ on time scales shorter than $\tau_{c}$. As shown below, however, the diffusion coefficients in both the anomalous and normal regimes explicitly depend on $\alpha$. Note that also for finite-size LWs finite $T$ effects of trajectories are relevant [22].

\section{B. Linear response theory}

We now turn to the linear response of an LW to a small external perturbation $H_{p}=-x f(t)$, where $f(t)$ may be explicitly time dependent. Similar to the independence of the waiting time density $\psi(t)$ of an external force field of uncoupled CTRW processes [8,29], a biased LW still switches between $\pm v$, albeit asymmetrically [30]. With the characteristic time scale $\tau_{c}$, the diffusivity converges, $D\left(\alpha, \tau_{c}\right)=\lim _{t \rightarrow \infty} \int_{0}^{t} C_{v}\left(t^{\prime}\right) d t^{\prime}<\infty$, so that we may apply linear response theory. Note that the general problem of linear response in superdiffusion remains open, and thus the study case of LWs is even more relevant [31]. 

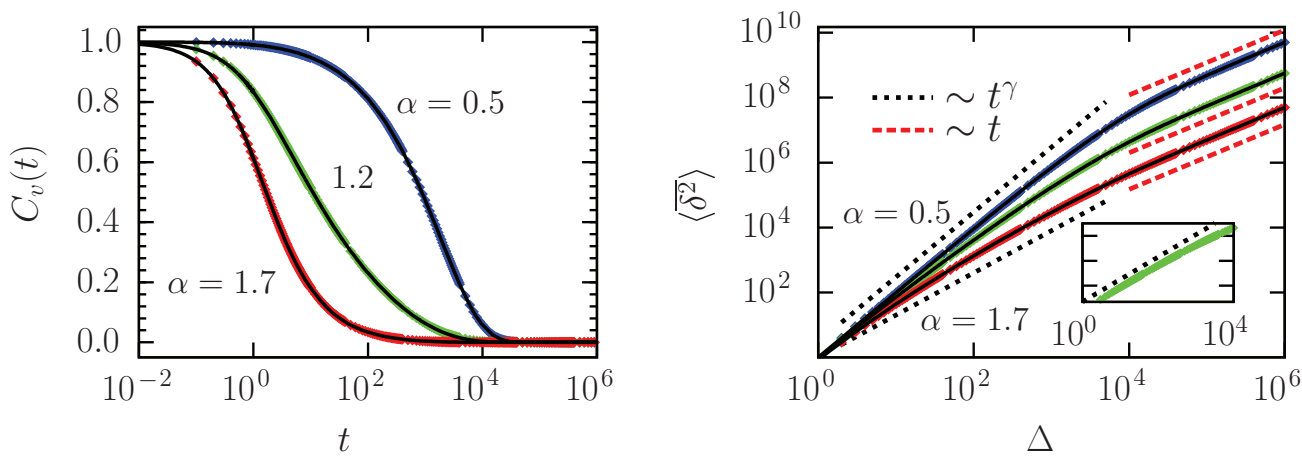

FIG. 1. (Color online) Time evolution of the velocity autocorrelation $C_{v}(t)$ (left) and time-averaged MSD $\overline{\delta x^{2}}$ (right). Symbols represent simulations results, full lines are evaluated from Eqs. (3) and (5). Dashed lines depict the asymptotic scaling. Results are shown for $\alpha=0.5$, 1.2, and 1.7, as indicated. The simulation results were obtained from ensembles of $10^{4}$ trajectories of length $10^{8} \Delta_{1}$, and $\tau_{c}=10^{4}$ (used in all graphs).

Applying linear response theory for given $H_{p}$, we obtain the perturbed velocity

$$
\langle v(t)\rangle_{p} \equiv \frac{d}{d t}\langle x(t)\rangle_{p}=\int_{-\infty}^{t} \beta C_{v}\left(t-t^{\prime}\right) f\left(t^{\prime}\right) d t^{\prime},
$$

where $\beta=1 /\left(k_{B} \mathcal{T}\right)$ is the inverse thermal energy. Consider first a constant force $f$ switched on at $t=0$. With the velocity autocorrelation (3) the average velocity in the perturbed ensemble becomes

$$
\langle v(t)\rangle_{p}=\frac{\beta f}{\mathcal{N}}\left[\sum_{k=0}^{2} \frac{(-1)^{k+1}}{2^{\delta_{k, 1}-1} k !} \Gamma\left(\alpha, \frac{1+t}{\tau_{c}}\right)+\frac{\Lambda\left(\alpha, \tau_{c}\right)}{2}\right],
$$

where we use the abbreviation

$$
\mathcal{N}=\Gamma\left(1-\alpha, \tau_{c}^{-1}\right)-\tau_{c}^{-1} \Gamma\left(-\alpha, \tau_{c}^{-1}\right),
$$

and where $\delta_{k, 1}$ is the Kronecker symbol. Moreover, the parameter $\Lambda$ is defined through

$$
\begin{aligned}
\Lambda\left(\alpha, \tau_{c}\right)= & \Gamma\left(-\alpha, \tau_{c}^{-1}\right)\left[2 \alpha \tau_{c}^{-1}+\alpha(\alpha-1)+\tau_{c}^{-2}\right] \\
& +\tau_{c}^{\alpha} e^{-1 / \tau_{c}}\left(1-\alpha-\tau_{c}^{-1}\right) .
\end{aligned}
$$

The instantaneous response irrespective of $\alpha$ is linear for the velocity, $\langle v(t)\rangle_{p} \sim t$, corresponding to the ballistic initial dynamics. At intermediate times $1 \ll t \ll \tau_{c}$ we find

$$
\langle v(t)\rangle_{p} \sim \beta f \begin{cases}t, & 0<\alpha<1, \\ \frac{1}{2-\alpha} t^{2-\alpha}, & 1<\alpha<2,\end{cases}
$$

i.e., a nonlinear anomalous drift

$$
\langle x(t)\rangle_{p} \sim \beta f \begin{cases}\frac{1}{2} t^{2}, & 0<\alpha<1, \\ \frac{1}{(2-\alpha)} t^{3-\alpha}, & 1<\alpha<2 .\end{cases}
$$

In analogy to the unperturbed MSD, $\langle x(t)\rangle_{p}$ scales ballistically for $0<\alpha<1$ and subballistically for $1<\alpha<2$ (Fig. 2). We stress that the average velocity in the perturbed (stationary) ensemble is time dependent and thus different from the case of perturbed maps [32]. In the limit $t \gg \tau_{c}$ we find the constant drift velocity $\langle v(t)\rangle_{p}=\beta f D\left(\alpha, \tau_{c}\right)$,

$$
D\left(\alpha, \tau_{c}\right)=\frac{1}{2 \tau_{c} \mathcal{N}} \sum_{k=0}^{2} 2^{\delta_{k, 1}}\left(-\tau_{c}\right)^{k} \Gamma\left(k-\alpha, \tau_{c}^{-1}\right),
$$

compare to Fig. 3. With the Kubo formula we then recover the Einstein relation

$$
\langle x(t)\rangle_{p}=\frac{1}{2} \beta f\left\langle x^{2}(t)\right\rangle_{s}
$$

between the drift $\langle x(t)\rangle_{p}$ and the stationary $\operatorname{MSD}\left\langle x^{2}(t)\right\rangle_{s}$, valid for all $t$. As a transient, this includes the generalized Einstein relation [33] with the nonlinear drift $\langle x(t)\rangle_{p} \sim K_{\mu} t^{\mu}$

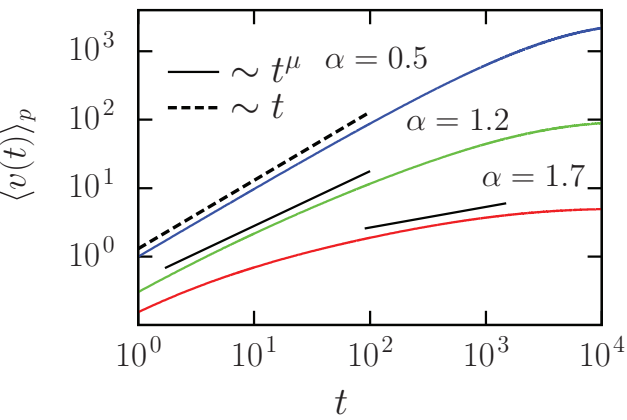

FIG. 2. (Color online) (Left) Drift velocity $\langle v(t)\rangle_{p}$ normalized to $\beta f D\left(\alpha, \tau_{c}\right)$ for $\alpha=0.5,1.2$, and 1.7. Symbols: Numerical integration of $\beta f C_{v}(t)$. Full lines: Exact analytical solution (11). (Right) Analytical solution (11) in units of $\beta f$ with intermediate-time scaling. For visual convenience the cases $\alpha=1.2$ and 1.7 were scaled by a factor of $1 / 3$ and $1 / 5$, respectively. 

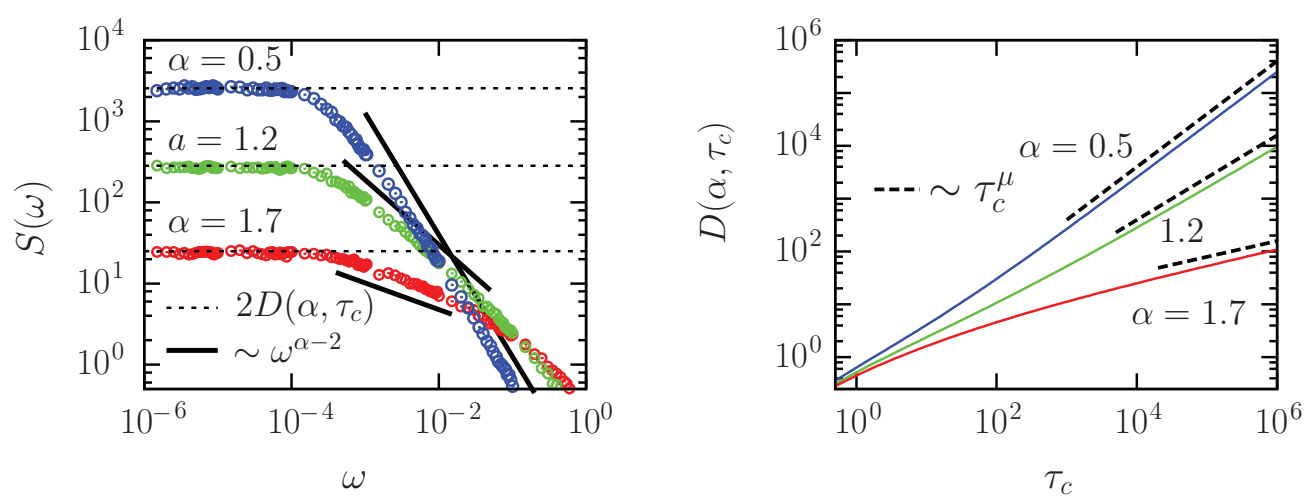

FIG. 3. (Color online) (Left) Power spectrum of velocity fluctuations for $\alpha=0.5,1.2$, and 1.7. Symbols: Simulation results. Lines: Expected scaling behavior, see text. (Right) Long time diffusivity $D\left(\alpha, \tau_{c}\right)$ versus $\tau_{c}$ for the same $\alpha$ values. Lines: Eq. (16). Dashed lines: asymptotic scaling, Eq. (30).

$(\mu \neq 1)$. Below we will see the alternative Einstein relation in the nonstationary ensemble. Figure 2 shows excellent agreement between the numerical integration of the velocity autocorrelation and Eq. (14). In Fig. 2, for the normalized drift velocity the response delay is shortest for the subballistic case $1<\alpha<2$ and increases towards smaller $\alpha$ values. As at long times the drift is proportional to $D\left(\alpha, \tau_{c}\right)$, however, the absolute drift is stronger for smaller $\alpha$ (Fig. 3). Figure 2 also shows the multiscale nature of $\langle v(t)\rangle_{p}$ at larger $\alpha$, while the linear scaling for $\alpha=0.5$ is nicely fulfilled.

\section{Power spectrum}

We now consider a periodic perturbation $f \cos (\omega t)$, i.e., we study the power spectrum of the velocity fluctuations

$$
S(\omega)=\lim _{T \rightarrow \infty}(2 T)^{-1}\left|\int_{0}^{T} v(t) \exp (-i \omega t) d t\right|^{2},
$$

which can be related to the response via the fluctuationdissipation theorem

$$
\langle v(t)\rangle_{p}=\beta f \operatorname{Re}\left\{S(\omega) e^{i \omega t}\right\}
$$

We calculate $S(\omega)$ from the Laplace transform of $C_{v}(t)$ via

$$
S(\omega)=\lim _{\eta \rightarrow 0}\left[\tilde{C}_{v}(u=\eta+i \omega)+\tilde{C}_{v}(u=\eta-i \omega)\right]
$$

The quantity $\tilde{C}_{v}(u)$ is in turn related to the Laplace transform of the renewal expression

$$
\tilde{C}_{v}(u)=u^{-1}+\bar{\tau} u^{-2}(1-\tilde{\psi}(u)) .
$$

The Laplace transform of $\psi(t), \mathcal{L}\{\psi(t)\}$, reads

$$
\tilde{\psi}(u)=\frac{\tau_{c}^{\alpha} \exp (u)}{\Gamma\left(-\alpha, \tau_{c}^{-1}\right)}\left(u+\tau_{c}^{-1}\right)^{\alpha} \Gamma\left(-\alpha, u+\tau_{c}^{-1}\right) .
$$

Depending on the scale of $u$ relative to $\tau_{c}^{-1}$, it can be shown that $\tilde{\psi}(u)$ has two different expansions given in Appendix A.
For $\tau_{c}^{-1} \ll u \ll 1 \tilde{\psi}(u)$ behaves as

$$
\tilde{\psi}(u) \sim 1+\frac{\mathcal{P} u^{\alpha}}{2}\left(1+\frac{\alpha}{\tau_{c} u}\right)+\left[\mathcal{B}\left(\alpha, \tau_{c}\right)-\bar{\tau}\right] u,
$$

where we have introduced

$$
\mathcal{P}=\frac{\tau_{c}^{\alpha} \Gamma(-\alpha)}{\Gamma\left(-\alpha, \tau_{c}^{-1}\right)},
$$

with the asymptotic behavior

$$
\mathcal{P} \sim \frac{\Gamma(-\alpha) \alpha(1-\alpha)}{1-\alpha\left(1-\tau_{c}^{-1}\right)}
$$

as $\tau_{c} \rightarrow \infty$, and

$$
\mathcal{B}\left(\alpha, \tau_{c}\right)=\frac{\tau_{c} \Gamma(1-\alpha)}{\Gamma\left(-\alpha, \tau_{c}^{-1}\right)}\left(1+\frac{1}{\alpha \tau_{c}}\right),
$$

which in the limit $\tau_{c} \rightarrow \infty$ behaves as $\mathcal{B}\left(\alpha, \tau_{c}\right) \sim \bar{\tau}$ for $0<$ $\alpha<1$ and as $\mathcal{B}\left(\alpha, \tau_{c}\right) \sim \tau_{c}^{1-\alpha} \Gamma(2-\alpha) /\left[1-\alpha\left(1-\tau_{c}^{-1}\right)\right]$ for $1<\alpha<2$. In the regime $u \ll \tau_{c}^{-1} \tilde{\psi}(u)$ has the expansion

$$
\tilde{\psi}(u) \sim 1-\bar{\tau} u+\bar{\tau} D\left(\alpha, \tau_{c}\right) u^{2} .
$$

Using the expressions in $\tilde{C}_{v}(u)$ from above, we obtain the power spectrum given in Eq. (A2) of the Appendix. The exact expressions contain alternating and oscillating series, which are quite difficult to evaluate numerically. We therefore focus on the asymptotic behavior. In the range $\tau_{c}^{-1} \ll \omega \ll 1$, we find

$$
S(\omega) \sim 2 \xi_{\alpha} \omega^{\alpha-2}\left[-\cos \left(\frac{\alpha \pi}{2}\right)+\frac{\alpha}{\omega \tau_{c}} \sin \left(\frac{\alpha \pi}{2}\right)\right],
$$

where

$$
\xi_{\alpha}= \begin{cases}-\tau_{c}^{\alpha-1} /\left(\alpha+\tau_{c}^{-1}\right), & 0<\alpha<1, \\ \Gamma(2-\alpha), & 1<\alpha<2\end{cases}
$$

In the limit $\omega \ll \tau_{c}^{-1}$ we have $S(\omega) \sim 2 D\left(\alpha, \tau_{c}\right)$, the required convergence to the long time diffusion coefficient. Figure 3 
shows our asymptotic analytical forms of $S(\omega)$ together with LW simulations and the direct transform of $v(t)$. The LW behavior is thus a direct generalization of the Lorentzian shape of the power spectrum of a random telegraph process, equivalent to an LW with exponential $\psi(t)$.

Moreover, it can be shown that $D\left(\alpha, \tau_{c}\right)$ has the following asymptotic behavior in the regime $\tau_{c} \rightarrow \infty$,

$$
D\left(\alpha, \tau_{c}\right) \sim \frac{|1-\alpha|}{2} \begin{cases}\tau_{c}, & 0<\alpha<1, \\ \alpha \Gamma(2-\alpha) \tau_{c}^{2-\alpha}, & 1<\alpha<2 .\end{cases}
$$

This rigorous result can also be obtained from scaling arguments: the characteristic time scale of the intermediate asymptotic dynamics is $\tau_{c}$. From Eq. (9), $\left\langle x^{2}\left(\tau_{c}\right)\right\rangle_{s} \sim \tau_{c}^{\gamma}$, with $\gamma=2$ for $0<\alpha<1$ and $\gamma=3-\alpha$ for $1<\alpha<2$. Hence we find $D\left(\alpha, \tau_{c}\right) \simeq \tau_{c}^{\gamma-1}$ (Fig. 3): in unbounded systems, the diffusivity diverges with the system size.

The fluctuation-dissipation relation for LWs is thus

$$
S(\omega=0)=2 D\left(\alpha, \tau_{c}\right) \sim \tau_{c}^{\min (1,2-\alpha)} .
$$

Thus, in superdiffusive systems $S(\omega=0)$ diverges at most linearly with the system size $L \simeq \tau_{c}$, such that for finite (but otherwise arbitrarily large) $L$ the classical fluctuationdissipation theorem holds rigorously, without limitations. Moreover, as noted already in Ref. [5] a scaling $S(\omega) \sim \omega^{\alpha-2}$ extending to zero frequency for $0<\alpha<1$ as expected from $\psi(t)$ without regularization cannot be physical, as it would imply a divergence of $C_{v}(t=0)$, which by definition must be finite and proportional to $\left\langle v^{2}\right\rangle$. In Refs. [5,21,28] this incompatibility was avoided by assuming a low-frequency cutoff.

\section{Ergodic properties and Einstein relations}

We now address the ergodic properties of finite-size LWs. A necessary requirement for ergodicity is that $\lim _{t \rightarrow \infty} \frac{1}{t} \int_{0}^{t} C_{v}\left(t^{\prime}\right) d t^{\prime}=0[34,35]$. We evaluated this integral in our derivation of Eq. (14), from which we find for $t, \tau_{c} \rightarrow \infty$ but $t \ll \tau_{c}$ that the dynamics on intermediate time scales is nonergodic for $0<\alpha<1$ as the integral tends to unity, see also Refs. [22,23]. For $1<\alpha<2$ the integral converges to zero, formally indicating ergodicity. However, as shown previously $[3,22,23]$ this regime features an ultraweak ergodicity breaking, see below.

As mentioned, the time-averaged MSD $\overline{\delta^{2}(\Delta)}$ equals the ensemble MSD in the stationary state, $\left\langle x^{2}(t)\right\rangle_{s}$ of a system prepared at $t=-\infty$, and thus $\overline{\delta^{2}(\Delta)}$ and $\left\langle x^{2}(t)\right\rangle_{s}$ are independent of the initial condition. In contrast, the ensemble averaged $\operatorname{MSD}\left\langle x^{2}(t)\right\rangle_{n s}$ in the nonstationary state refers to initiation at $t=0$. Both conditions are experimentally relevant. In both cases the observation is assumed to start at $t=0$. For LWs a stationary state exists for $1<\alpha<2$ or, for any $0<\alpha<2$, if the power-law shape of $\psi(t)$ is regularized at the time scale $\tau_{c}$.

To calculate $\overline{\delta^{2}(\Delta)}$ we use the CTRW approach [28], yielding the Laplace transform $\overline{\delta^{2}(u)}=2 u^{-3}-2 u^{-4}(1-\tilde{\psi}(u)) / \bar{\tau}$. In the intermediate, anomalous regime $1 \ll \Delta, t \ll \tau_{c}$ the following leading order behaviors of $\overline{\delta^{2}(\Delta)}$ emerge:

$$
\overline{\delta^{2}(\Delta)} \sim \Delta^{2}\left(1-\frac{2\left(\Delta / \tau_{c}\right)^{1-\alpha}}{\left(\alpha+\tau_{c}^{-1}\right) \Gamma(4-\alpha)}\right)
$$

for $0<\alpha<1$. At $\tau_{c} \rightarrow \infty$ we recover the ballistic scaling for infinite trajectories [23,28,32]. For $1<\alpha<2$,

$$
\overline{\delta^{2}(\Delta)} \sim \frac{2 \Delta^{3-\alpha}}{\zeta}\left(1-\frac{1}{2}\left(\alpha+\tau_{c}^{-1}\right) \Gamma(4-\alpha)\left[\frac{\Delta}{\tau_{c}}\right]^{\alpha-1}\right),
$$

and $\overline{\delta^{2}(\Delta)} \sim 2 \Delta^{3-\alpha} / \zeta$ for $\tau_{c} \rightarrow \infty[22,23,28]$.

In the nonstationary ensemble the CTRW approach yields $\left\langle x^{2}(u)\right\rangle_{n s}=2\left(1-\tilde{\psi}(u)+u \partial_{u} \tilde{\psi}(u)\right) /\left(u^{3}(1-\tilde{\psi}(u))\right.$. It can be shown that in the range $u \geqslant \tau_{c}^{-1}$ Eqs. (A3) in Appendix A have the following behavior for small $u$ :

$$
\partial_{u} \tilde{\psi}(u) \sim\left[\mathcal{B}\left(\alpha, \tau_{c}\right)-\bar{\tau}\right]+\frac{\alpha}{2} \mathcal{P} u^{\alpha-1}\left(1+\frac{\alpha-1}{\tau_{c} u}\right),
$$

when $\tau_{c}^{-1} \ll u \ll 1$ and

$$
\partial_{u} \tilde{\psi}(u) \sim-\bar{\tau}+2 \bar{\tau} D\left(\alpha, \tau_{c}\right) u,
$$

when $u \ll \tau_{c}^{-1}$. Using Eqs. (33) in the expression for $\left\langle x^{2}(u)\right\rangle_{n s}$ we find

$$
\left\langle x^{2}(t)\right\rangle_{n s} \sim t^{2}(1-\alpha)\left(1-\frac{\alpha}{3(1-\alpha)} \frac{t}{\tau_{c}}\right),
$$

in the range $0<\alpha<1$, while for $1<\alpha<2$ we arrive at

$\left\langle x^{2}(t)\right\rangle_{n s} \sim \frac{2 t^{3-\alpha}}{\zeta}(\alpha-1)\left(1-\frac{\alpha(2-\alpha)}{(4-\alpha)(\alpha-1)} \frac{t}{\tau_{c}}\right)$.

Equations (32) and (34) determine the ergodicity breaking parameter $\mathcal{E B}(t)=\overline{\delta^{2}(t)} /\left\langle x^{2}(t)\right\rangle_{n s}$, which for $t \ll \tau_{c}$ can be written compactly as $[22,36]$

$$
\mathcal{E B}(t) \sim \frac{1}{|1-\alpha|} \frac{1-A_{1}(\alpha)\left(t / \tau_{c}\right)^{|1-\alpha|}}{1-A_{2}(\alpha)\left(t / \tau_{c}\right)},
$$

where $A_{1}(\alpha)$ and $A_{2}(\alpha)$ are defined as

$$
A_{1}(\alpha)= \begin{cases}2 /\left[\left(\alpha+\tau_{c}^{-1}\right) \Gamma(4-\alpha)\right], & 0<\alpha<1, \\ \left(\alpha+\tau_{c}^{-1}\right) \Gamma(4-\alpha) / 2, & 1<\alpha<2,\end{cases}
$$

and

$$
A_{2}(\alpha)= \begin{cases}\alpha(2 \alpha-1) /[3(\alpha-1)], & 0<\alpha<1 \\ \alpha(2-\alpha) /[(4-\alpha)(\alpha-1)], & 1<\alpha<2 .\end{cases}
$$

From Eq. (35) we observe that the regularization at $\tau_{c}$ affects $\left\langle x^{2}(t)\right\rangle_{n s}$ more strongly than $\overline{\delta^{2}}$, which is a natural consequence of the sliding time average. In the limit $\tau_{c} \rightarrow \infty$ we recover the ultraweak ergodicity breaking $\mathcal{E B}(t) \sim 1 /|1-\alpha|[22,23,28]$. Conversely, from the asymptotic forms for $u \ll \tau_{c}^{-1}$ we find that on time scales $t \gg \tau_{c}$ the system is ergodic, $\mathcal{E B}(t)=1$. The regularization of $\psi(t)$ at the characteristic time $\tau_{c}$ introduces a natural time scale, beyond which ergodicity is restored.

The nonstationary MSD $\left\langle x^{2}(t)\right\rangle_{n s}$ depends on the initial condition. Hence, there exists another variant of the Einstein 
relation. This nonstationary Einstein relation reads

$$
\langle x(t)\rangle_{p}^{n s}=\frac{1}{2} \beta f\left\langle x^{2}(t)\right\rangle_{n s}=\frac{1}{2} \beta f \kappa(t) \overline{\delta^{2}(t)},
$$

where $\kappa(t)=1$ for $t \gg \tau_{c}$ and $\kappa(t)=1 / \mathcal{E} \mathcal{B}(t)$ for $1 \ll t \leqslant \tau_{c}$. The stationary form (17) thus differs from the nonstationary one here by the factor $\kappa(t)$. For $\tau_{c} \rightarrow \infty$ a similar relation was reported in Ref. [33]. We can also obtain an exact expression for the first moment in the presence of s small perturbation averaged over a time $T$,

$$
\langle\overline{\delta(\Delta)}\rangle_{p}=\frac{1}{T-\Delta} \int_{0}^{T-\Delta}\left[\langle x(t+\Delta)\rangle_{p}-\langle x(t)\rangle_{p}\right] d t,
$$

where the angular brackets denote an average over many trajectories, $\langle\overline{\delta(\Delta)}\rangle=N^{-1} \sum_{i=1}^{N} \overline{\delta_{i}(\Delta)}$. The detailed calculation and exact result will be presented in a separate work [37], here we merely present the intermediate-time and long-time asymptotic results. In the regime $1 \ll T-\Delta \ll \tau_{c}$ we obtain in the stationary ensemble

$$
\langle\overline{\delta(\Delta)}\rangle_{p}^{s} \sim \frac{\beta f T \Delta}{2}\left(1-\frac{6\left(T / \tau_{c}\right)^{1-\alpha}}{\left(\alpha+\tau_{c}^{-1}\right) \Gamma(5-\alpha)}\right)
$$

for $0<\alpha<1$ and

$$
\langle\overline{\delta(\Delta)}\rangle_{p}^{s} \sim \frac{\beta f T^{2-\alpha} \Delta}{\zeta}\left(1-\frac{\left(\alpha+\tau_{c}^{-1}\right) \Gamma(5-\alpha)}{6}\left[\frac{T}{\tau_{c}}\right]^{\alpha-1}\right)
$$

for $1<\alpha<2$. For the same regime in the nonstationary ensemble we arrive at

$$
\langle\overline{\delta(\Delta)}\rangle_{p}^{n s} \sim(1-\alpha) \frac{\beta f T \Delta}{2}\left(1-\frac{\alpha\left(T / \tau_{c}\right)}{4(1-\alpha)}\right)
$$

for $0<\alpha<1$ and

$$
\langle\overline{\delta(\Delta)}\rangle_{p}^{n s} \sim(\alpha-1) \frac{\beta f T^{2-\alpha} \Delta}{\zeta}\left(1-\frac{\alpha(2-\alpha)}{(5-\alpha)(\alpha-1)} \frac{T}{\tau_{c}}\right),
$$

for $1<\alpha<2$. If, on the other hand, $T-\Delta \gg \tau_{c}$ we recover in both ensembles the universal result

$$
\langle\overline{\delta(\Delta)}\rangle_{p} \sim \frac{\beta f}{2} D\left(\alpha, \tau_{c}\right) \Delta
$$

which is, most importantly, independent of $T$. In all cases we find a linear scaling of $\langle\bar{\delta}\rangle_{p}$ with the time lag $\Delta$. Moreover, we find that just as ergodicity is restored on time scales $t \gg \tau_{c}$, a time-averaged Einstein relation

$$
\langle\overline{\delta(\Delta)}\rangle_{p}=\frac{\beta f}{2}\left\langle\overline{\delta^{2}(\Delta)}\right\rangle
$$

holds as well, irrespective of the choice of the ensemble. The Einstein relations are valid for sufficiently small magnitudes of the driving force $f$ such that the system does not exceed a maximum velocity corresponding to the scale $\sqrt{k_{B} T_{\text {eff }} / m}$ set by some effective temperature $\mathcal{T}_{\text {eff }}$ that corresponds to the overall energy stored in the system, and nonlinear effects can be neglected. At $\tau_{c} \rightarrow \infty$ we formally recover the scaling reported in Ref. [23]. However, as already noted by the authors of Ref. [33], in the absence of a cutoff time the corresponding Einstein relation has an "expiration date" (for an arbitrarily weak bias the Einstein relation will break down eventually). This means that in the absence of a cutoff time (e.g., in an infinite system) the range of validity of the cutoff free analogues of Eqs. (40) with respect to the magnitude of $f$, as applied by the authors of Ref. [23], is in fact not defined, but depends additionally on both $T$ and $\Delta$. Hence, for extremely long trajectories, i.e., $T \rightarrow \infty$, the relations strictly only hold for $f=0$. In contrast, when $\psi(t)$ is regularized at $\tau_{c}$, the linear response theory for both ensemble- and timeaveraged quantities has a well-defined range of validity and rigorously recovers the time-averaged Einstein relation (42) for $\Delta \gg \tau_{c}$.

\section{CONCLUSION}

Concluding, we investigated the physical properties of finite-size LWs whose power-law waiting time density is regularized by a characteristic time scale $\tau_{c}$ proportional to the physical system size $L$. Finite-size LWs thus allow us to exactly apply the Kubo linear response theory. In the presence of a constant, weak external force we found that the Einstein relation holds at all times and for all values of $\alpha$. In particular, we discussed both the stationary and nonstationary cases. At short times the MSD is ballistic, at intermediate times it follows the classical, anomalous LW scaling, and beyond $\tau_{c}$ the MSD is linear in time. For a weak periodic forcing we obtained the scaling of the spectrum at high frequencies. At low frequencies it turns over to a plateau whose value is the diffusion coefficient of the normally diffusive long time behavior at $t \gg \tau_{c}$, and thus provides the fluctuation-dissipation relation for finite-size LWs. This diffusion constant explicitly depends on $\tau_{c}$ and $\alpha$. Finite-size LWs provide a complete physical picture for superdiffusive, physical systems. Due to the time scale $\tau_{c}$ classical response theory can be applied, without resorting to the introduction of artificial cutoffs. In that sense our present approach expands on relevant previous works on LWs $[3,5,21,23,30]$.

Physically, these observations correspond to the fact that when $t \rightarrow \tau_{c}$ the anomalous response gradually turns normal, and the induced velocity never exceeds the thermal velocity. To cure the diverging linear response behavior in superdiffusive systems without truncation, for deterministic chaotic systems it was suggested to introduce a relaxation time $t_{c}$ and split the velocity correlation $C_{v}$ into two parts [31]. The anomalous part of $C_{v}$ ranges up to $t_{c}$, beyond which the normal regime takes over. The velocity correlation $C_{v}(t)$ discussed in the present work inherently possesses such properties and does not demand an ad hoc separation of the integral.

We analyzed the ergodic properties of LWs in terms of the stationary ensemble averaged MSD $\left\langle x^{2}(t)\right\rangle$ (equal to $\overline{\delta^{2}}$ ) and the nonstationary MSD $\left\langle x^{2}(t)\right\rangle_{n s}$. Consistently with the linear response behavior we find an ultraweak ergodicity breaking at intermediate times, such that $\overline{\delta^{2}(t)}$ and $\left\langle x^{2}(t)\right\rangle_{n s}$ differ by a constant, which depends on $\alpha$ and the ratio of lag time $\Delta$ (or regular time $t$ ) and $\tau_{c}$. When $\tau_{c} \rightarrow \infty$, this factor reduces to the known value $1 /|1-\alpha|$, while for times beyond $\tau_{c}$ the system is ergodic.

LWs naturally extend random walk processes to the superdiffusive regime. For subdiffusive CTRW processes, 
both the linear response $[29,38]$ and the weakly nonergodic properties $[39,40]$ were extensively discussed and verified experimentally, and the ageing behavior analyzed [41]. It will be interesting to study whether the results obtained here are indeed observed in experiments, and how (transient) ageing affects the system response. Another question of interest is whether the current results can be extended to more general superdiffusive systems.

\section{ACKNOWLEDGMENTS}

We acknowledge funding from the Academy of Finland (FiDiPro scheme) and the German Federal Ministry for Education and Research.

\section{APPENDIX A: EXPANSION OF THE LAPLACE TRANSFORM OF $\tilde{\psi}(u)$}

In the regime $u \geqslant \tau_{c}^{-1}$ we obtain

$$
\begin{aligned}
\tilde{\psi}(u)= & \frac{\tau_{c}^{\alpha} \Gamma(-\alpha)}{\Gamma\left(-\alpha, \tau_{c}^{-1}\right)} \sum_{k, l=0}^{\infty}\left[\left(\begin{array}{l}
\alpha \\
l
\end{array}\right) \frac{\tau_{c}^{-l}}{k !} u^{k-l+\alpha}\right. \\
& \left.-\sum_{m=0}^{l}\left(\begin{array}{c}
l \\
m
\end{array}\right) \frac{(-1)^{l} \tau_{c}^{-m}}{\Gamma(-\alpha)(l-\alpha) k ! l !} u^{k+l-m}\right] .
\end{aligned}
$$

For $u<\tau_{c}^{-1}$ we find instead

$$
\begin{aligned}
\tilde{\psi}(u)= & \frac{\tau_{c}^{\alpha} \Gamma(-\alpha)}{\Gamma\left(-\alpha, \tau_{c}^{-1}\right)} \sum_{k, l=0}^{\infty}\left[\left(\begin{array}{l}
\alpha \\
l
\end{array}\right) \frac{\tau_{c}^{l-\alpha}}{k !} u^{k+l}\right. \\
& \left.-\sum_{m=0}^{l}\left(\begin{array}{l}
l \\
m
\end{array}\right) \frac{(-1)^{l} \tau_{c}^{-m}}{\Gamma(-\alpha)(l-\alpha) k ! l !} u^{k+l-m}\right] .
\end{aligned}
$$

The corresponding expansion for the derivatives $\partial_{u} \tilde{\psi}(u)$ are

$$
\begin{aligned}
\partial_{u} \tilde{\psi}(u)= & \frac{\tau_{c}^{\alpha} \Gamma(-\alpha)}{\Gamma\left(-\alpha, \tau_{c}^{-1}\right)} \\
& \times\left[\sum_{k, l=0}^{\infty} \frac{\tau_{c}^{-l} u^{k-l+\alpha-1}}{k !}\left\{u\left(\begin{array}{l}
\alpha \\
l
\end{array}\right)-\alpha\left(\begin{array}{c}
\alpha-1 \\
l
\end{array}\right)\right\}\right. \\
& -\sum_{m=0}^{l}\left(\begin{array}{l}
l \\
m
\end{array}\right) \frac{(-1)^{l} \tau_{c}^{-m}}{\Gamma(-\alpha) k ! l !} u^{k+l-m} \\
& \left.\times\left\{\frac{1}{l-\alpha}-\frac{1}{l+1-\alpha}\right\}\right]
\end{aligned}
$$

whereas for $u<\tau_{c}^{-1}$ we instead obtain

$$
\begin{aligned}
\partial_{u} \tilde{\psi}(u)= & \frac{\tau_{c}^{\alpha} \Gamma(-\alpha)}{\Gamma\left(-\alpha, \tau_{c}^{-1}\right)} \\
& \times\left[\sum_{k, l=0}^{\infty} \frac{\tau_{c}^{l+1-\alpha} u^{k+l}}{k !}\left\{\tau_{c}^{-1}\left(\begin{array}{l}
\alpha \\
l
\end{array}\right)-\alpha\left(\begin{array}{c}
\alpha-1 \\
l
\end{array}\right)\right\}\right. \\
& -\sum_{m=0}^{l}\left(\begin{array}{l}
l \\
m
\end{array}\right) \frac{(-1)^{l} \tau_{c}^{-m}}{\Gamma(-\alpha) k ! l !} u^{k+l-m} \\
& \left.\times\left\{\frac{1}{l-\alpha}-\frac{1}{l+1-\alpha}\right\}\right]
\end{aligned}
$$

\section{APPENDIX B: FLUCTUATION POWER SPECTRUM}

In the regime $\omega \geqslant \tau_{c}^{-1}$ we obtain

$$
\begin{aligned}
S(\omega)= & \frac{2 \tau_{c}^{\alpha} \Gamma(-\alpha)}{\bar{\tau} \Gamma\left(-\alpha, \tau_{c}^{-1}\right)} \sum_{k, l=0}^{\infty} \\
& \times\left[a_{k l} \omega^{k-l+\alpha-2}-\sum_{m=0}^{l} b_{k l m} \omega^{k+l-m}\right],
\end{aligned}
$$

where the coefficients are given by

$$
\begin{aligned}
a_{k l}= & \left(\begin{array}{c}
\alpha \\
l
\end{array}\right) \frac{\tau_{c}^{-l}}{k !} \cos \left([k-l+\alpha-2] \frac{\pi}{2}\right), \\
b_{k l m}= & \Gamma(-\alpha)^{-1}\left(\begin{array}{c}
l \\
m
\end{array}\right) \frac{(-1)^{l} \tau_{c}^{-m}}{(l-\alpha) k ! l !} \cos \\
& \times\left([k+l-m] \frac{\pi}{2}\right)\left(1-\Delta_{l m}\right),
\end{aligned}
$$

with $\delta(\omega)$ and $\Delta_{l m}$ being the Dirac and Kronecker deltas, respectively. For $\omega<\tau_{c}^{-1}$ we get

$$
\begin{aligned}
S(\omega)= & \frac{2 \tau_{c}^{\alpha} \Gamma(-\alpha)}{\bar{\tau} \Gamma\left(-\alpha, \tau_{c}^{-1}\right)} \\
& \times \sum_{k, l=0}^{\infty}\left[c_{k l} \omega^{k+l}-\sum_{m=0}^{l} d_{k l m} \omega^{k+l-m}\right],
\end{aligned}
$$

with the expansion coefficients

$$
\begin{aligned}
c_{k l}= & \left(\begin{array}{c}
\alpha \\
l
\end{array}\right) \frac{\tau_{c}^{l-\alpha}}{k !} \cos \left([k+l] \frac{\pi}{2} \delta(\omega)\right)(\{1-\delta(\omega)\}), \\
d_{k l m}= & \Gamma(-\alpha)^{-1}\left(\begin{array}{c}
l \\
m
\end{array}\right) \frac{(-1)^{l} \tau_{c}^{-m}}{(l-\alpha) k ! l !} \cos \\
& \times\left([k+l-m] \frac{\pi}{2} \delta(\omega)\right)[1-\delta(k+l-m)] .
\end{aligned}
$$

[1] G. M. Zaslavsky, Hamiltonian Chaos and Fractional Dynamics (Oxford University Press, Oxford, England, 2008).

[2] J. Klafter, M. F. Shlesinger, and G. Zumofen, Phys. Today 49, 33 (1996).
[3] G. Zumofen and J. Klafter, Phys. Rev. E 47, 851 (1993); 51, 1818 (1995).

[4] T. H. Solomon, E. R. Weeks, and H. L. Swinney, Phys. Rev. Lett. 71, 3975 (1993). 
[5] T. Geisel and S. Thomae, Phys. Rev. Lett. 52, 1936 (1984); T. Geisel, J. Nierwetberg, and A. Zacherl, ibid. 54, 616 (1985).

[6] T. Akimoto and E. Barkai, Phys. Rev. E 87, 032915 (2013); E. Barkai and J. Klafter, Phys. Rev. Lett. 79, 2245 (1997).

[7] M. F. Shlesinger, J. Klafter, and Y. M. Wong, J. Stat. Phys. 27, 499 (1982).

[8] E. W. Montroll and G. H. Weiss, J. Math. Phys. 10, 753 (1969); H. Scher and E. W. Montroll, Phys. Rev. B 12, 2455 (1975).

[9] M. Magdziarz, W. Szczotka, and P. Żebrowski, J. Stat. Phys. 147, 74 (2012).

[10] In contrast to Lévy flights with diverging MSD [24].

[11] P. Barthelemy, J. Bertolotti, and D. S. Wiersma, Nature (London) 453, 495 (2008); P. Barthelemy, J. Bertolotti, K. Vynck, S. Lepri, and D. S. Wiersma, Phys. Rev. E 82, 011101 (2010).

[12] N. Mercadier, W. Guerin, M. Chevrollier, and R. Kaiser, Nat. Phys. 5, 602 (2009).

[13] C. Galland, Y. Ghosh, A. Steinbrück, M. Sykora, J. A. Hollingsworth, V. I. Klimov, and H. Htoon, Nature (London) 479, 203 (2011); F. D. Stefani, J. P. Hoogenboom, and E. Barkai, Phys. Today 62, 34 (2009).

[14] R. Nathan et al., Proc. Natl. Acad. Sci. USA 105, 19052 (2008).

[15] M. C. González, C. A. Hidalgo, and A.-L. Barabási, Nature (London) 453, 779 (2008); D. Brockmann, Phys. World 2, 31 (2010).

[16] D. W. Sims et al., Nature (London) 451, 1098 (2008); N. E. Humphries et al., ibid. 465, 1066 (2010).

[17] M. de Jager, F. J. Weissing, P. M. J. Herman, B. A. Nolet, and J. van de Koppel, Science 332, 1551 (2011).

[18] A. Mashanova, T. H. Oliver, and V. A. A. Jansen, J. R. Soc. Interface 7, 199 (2010).

[19] M. G. Lundy, A. Harrison, D. J. Buckley, E. S. Boston, D. D. Scott, E. C. Teeling, W. I. Montgomery, and J. D. R. Houghton, J. R. Soc. Interface 10, 20120489 (2012).

[20] M. A. Lomholt, T. Koren, R. Metzler, and J. Klafter, Proc. Natl. Acad. Sci. USA 105, 11055 (2008).

[21] M. Niemann, H. Kantz, and E. Barkai, Phys. Rev. Lett. 110, 140603 (2013).

[22] A. Godec and R. Metzler, Phys. Rev. Lett. 110, 020603 (2013).

[23] D. Froemberg and E. Barkai, Phys. Rev. E 87, 030104(R) (2013).
[24] J. Klafter, A. Blumen, and M. F. Shlesinger, Phys. Rev. A 35, 3081 (1987).

[25] To avoid the introduction of another symbol for a microscopic time scale, we symbolically use the value unity. The intermediate time range over which anomalous diffusion is observed is thus $1 \ll t \ll \tau_{c}$.

[26] In the following we set $v$ equal to unity.

[27] R. Kubo, J. Phys. Soc. Jpn. 12, 570 (1957); R. Kubo, M. Toda, and N. Hashitsume, Statistical Physics II: Nonequilibrium Statistical Mechanics (Springer, Heidelberg, Germany, 1985).

[28] G. Zumofen and J. Klafter, Physica D 69, 436 (1993).

[29] R. Metzler, E. Barkai, and J. Klafter, Europhys. Lett. 46, 431 (1999); Phys. Rev. Lett. 82, 3563 (1999).

[30] G. Trefán, E. Floriani, B. J. West, and P. Grigolini, Phys. Rev. E 50, 2564 (1994).

[31] U. Marini et al., Phys. Rep. 461, 111 (2008); G. Gradenigo, A. Sarracino, D. Villamaina, and A. Vulpiani, J. Stat. Mech. (2012) L06001.

[32] T. Akimoto, Phys. Rev. Lett. 108, 164101 (2012).

[33] E. Barkai and V. N. Fleurov, Phys. Rev. E 58, 1296 (1998).

[34] A. M. Yaglom, An Introduction to the Theory of Stationary Random Functions (Dover, New York, 1972).

[35] See also A. I. Khinchin, Mathematical Foundations of Statistical Mechanics (Dover, New York, 1949); S. Burov, R. Metzler, and E. Barkai, Proc. Natl. Acad. Sci. USA 107, 13228 (2010).

[36] To be distinguished from the ergodicity breaking parameter defined in Y. He, S. Burov, R. Metzler, and E. Barkai, Phys. Rev. Lett. 101, 058101 (2008).

[37] A. Godec and R. Metzler, (unpublished).

[38] F. Amblard, A. C. Maggs, B. Yurke, A. N. Pargellis, and S. Leibler, Phys. Rev. Lett. 77, 4470 (1996); E. Barkai and J. Klafter, ibid. 81, 1134 (1998).

[39] E. Barkai, Y. Garini, and R. Metzler, Phys. Today 65, 29 (2012).

[40] A. V. Weigel, B. Simon, M. M. Tamkun, and D. Krapf, Proc. Nat. Acad. Sci. USA 108, 6438 (2011); J.-H. Jeon, V. Tejedor, S. Burov, E. Barkai, C. Selhuber-Unkel, K. Berg-Sørensen, L. Oddershede, and R. Metzler, Phys. Rev. Lett. 106, 048103 (2011); G. Margolin, V. Protasenko, M. Kuno, and E. Barkai, Adv. Chem. Phys. 133, 327 (2006).

[41] E. Barkai, Phys. Rev. Lett. 90, 104101 (2003); J. H. P. Schulz, E. Barkai, and R. Metzler, ibid. 110, 020602 (2013). 